\title{
Economic Cycles and Urban Growth with Land Value and Land Rent
}

\author{
Wei-Bin Zhang \\ Ritsumeikan Asia Pacific University, Japan
}

\begin{abstract}
The purpose of this study is to examine the existence of business fluctuations in an urban growth model of endogenous wealth and land value with residential distribution over a linear city by Zhang (2017). Zhang synthesised the Solow growth model and the Alonso model. This paper generalises Zhang's model by allowing all the timeindependent parameters to be time-dependent. It examines the relationship between growth, urban structure and land value with different time-dependent exogenous shocks. We simulate the model to demonstrate the existence of equilibrium points, motion of the dynamic system, and oscillations due to different exogenous shocks. The paper makes a unique contribution to the literature of spatial economic growth by identifying cycles in a spatial growth model built on microeconomic foundation.
\end{abstract}

Keywords: Periodic shocks, business cycles, economic growth, national utility, land rent, land value, residential location

JEL classification: 041, 018, E21

\section{Introduction}

The purpose of this study is to study periodic economic changes due to different exogenous shocks in an urban growth model with endogenous wealth and land value. Although many studies have been carried out on identifying the existence of business cycles (Chiarella and Flaschel, 2000; Gandolfo, 2005; Lorenz, 1993; Puu, 2011; Shone, 2002; Zhang, 1991, 2005, 2006), there are only a few theoretical models which identify fluctuations due to dynamic interdependence between economic growth, wealth accumulation, and land value with residential structure. This study attempts to provide another contribution to the literature by identifying economic fluctuations in the urban economic growth model recently proposed by Zhang (2017).

Zhang (2017) built a growth model of a small city by synthesising the Solow growth model and Alonso urban residential model. The Solow model is the basic model in neoclassical growth theory and the Alonso model is the basic model in neoclassical

a College of International Management, Ritsumeikan Asia Pacific University, Jumonjibaru, Beppu-Shi, Oitaken, 874-8577 Japan. Email: wbz1@apu.ac.jp

\section{Acknowledgement}

* The author is grateful for the constructive comments of the anonymous referee and for the editorial help of the Production Editor Ivan Foo. 
urban economics. Zhang's study synthesises the Solow growth and Alonso residential models for describing a small city with residential location and land value. It deals with dynamic interactions between land value, land rent, wealth accumulation, amenity and change, residential distribution with exogenous rate of interest and utility level. It describes the economic production and growth mechanism on the basis of the Solow growth model (Solow, 1956). The modelling of residential distribution and spatial structure are based on the Alonso model (Alonso, 1964). The determination of the land value is based on Zhang's recent work on economic growth with endogenous land value (Zhang, 2017). The basic economic mechanisms of some main influential models could be synthesised in an integrated framework because the model applies an alternative approach to describing behaviour of households. The paper makes a unique contribution to the literature of spatial urban growth by identifying urban economic cycles with different exogenous shocks. The rest of the paper is organised as follows. Section 2 defines the spatial growth model with endogenous land value and residential location for a small-open economy. Section 3 simulates the motion of the economic system. Section 4 carries out comparative dynamic analysis with regard to exogenous changes in some parameters. Section 5 concludes the study. The appendix proves the main results in section 3 .

\section{The Model}

This section is built on Zhang (2017) by allowing all the time-independent parameters to be time-dependent. Except for adding time to the parameters, almost all aspects in this section is from Zhang (2017). The economy is an open urban economy built on a flat featureless plain. The system has one industrial sector (Solow, 1956). All workers reside in the city and work in the central business district (CBD). People travel only between dwelling sites and the CBD. An individual resides only at one location. The urban area consists of a finite strip of land extending from a fixed central business district with constant unit width. The city does not employ any worker who does not live in the city. The system is geographically linear and consists of two parts - the CBD and the residential area. We use $L$ to stand for the fixed (territory) length of the city. We assume that all economic activities are concentrated in the CBD. Let $\omega$ stand for the distance from the CBD to a point in the residential area. We use $R(t, \omega)$ and $p(t, \omega)$ to represent the land rent and land price at location $\omega$ at time $t$. Housing is measured by lot size. The total labour force is fully employed by the industrial sector. We select industrial good to serve as numeraire. As we assume that the transportation cost of workers to the city is dependent on the travel distance, land rent for housing should be spatially different.

\section{(a) The Total Labour Input}

We use $n(t, \omega)$ to denote the residential density at $\omega$. The population $N(t)$ is homogenous. The width of the urban area is assumed to be unity. According to the definition of $n(t, \omega)$, we have:

$$
N(t)=\int_{0}^{L} n(t, \omega) d \omega
$$




\section{(b) The Production Sector}

Let $F(t)$ and $K(t)$ stand for the production function and the total capital stock. The production function is specified as follows:

$$
F(t)=A(t) K^{\alpha(t)}(t) N^{\beta(t)}(t), \alpha(t), \beta(t)>0, \alpha(t)+\beta(t)=1
$$

where $A(t), \alpha(t)$, and $\beta(t)$ are positive parameters. As the city is small, the rate of interest $r^{*}(t)$ is determined by national markets. The wage rate $w(t)$ is determined by urban labour markets. The marginal conditions are given by:

$$
r^{*}(t)+\delta_{k}(t)=\frac{\alpha F(t)}{K(t)}, w(t)=\frac{\beta(t) F(t)}{N(t)}
$$

where $\delta_{k}(t)$ is the depreciation rate of physical capital. From (2) and (3) we solve:

$$
k^{*}(t) \equiv \frac{K(t)}{N(t)}=\left(\frac{\alpha(t) A(t)}{r^{*}(t)+\delta_{k}(t)}\right)^{1 / \beta(t)}, w(t)=\beta(t) A(t) k^{* \alpha(t)}(t)
$$

(c) The Relation between the Lot Size and Residential Density

Let us denote $I(t, \omega)$ as the lot size of the household at $\omega$. According to the definitions of $I(t, \omega)$ and $n(t, \omega)$, we have:

$$
n(t, \omega)=\frac{1}{l(t, \omega)}, \quad 0 \leq \omega \leq L
$$

\section{(d) Choice between Physical Wealth and Land}

Consider now a household with one unity of money. He can either invest in capital goods thereby earning a profit equal to the net own-rate of return $r^{*}(t)$ or invest in land thereby earning a profit equal to the net own-rate of return $R(t, \omega) / p(t, \omega)$. The two options are assumed to yield equal returns, i.e.

$$
\frac{R(t, \omega)}{p(t, \omega)}=r^{*}(t)
$$

\section{(e) Travel Time and Cost to the CBD}

A resident decides the time distribution between leisure time and travel time. Let $T_{0}(t)$ and $\Gamma(\omega)$ respectively stand for the total available time and the time spent on travelling between the residence and CBD. We have:

$$
T(\omega)+\Gamma(\omega, t)=T_{0}(t)
$$

where $T(\omega, t)$ is the leisure time that the household at $\omega$ enjoys. This study assumes that the travel cost $\mathrm{c}_{\mathrm{T}}(\omega, t)$ from location $\omega$ to the CBD is dependent on the distance as follows:

$$
c_{T}(t, \omega)=\bar{c}(t)+c_{0}(\omega, t)
$$




\section{(f) Land Ownership, Current Income and Disposable Income}

Let $\bar{k}(\omega, t)$ stand for the representative household's physical wealth, and $a(\omega, t)$ for the value of land owned by the household at location $\omega$. For simplicity we assume that all the land is owned by the city's population. The total value of land owned by the household at $\omega$ is the sum of all the value of land the household owns in the economy. We have:

$$
a(t, \omega)=\int_{0}^{L} p(t, \widetilde{\omega}) \bar{l}(t, \omega, \widetilde{\omega}) d \widetilde{\omega}
$$

where $\bar{I}(t, \omega, \tilde{\omega})$ is the land that the household at $\omega$ owns at $\tilde{\omega}$. The total value of wealth $v(t, \omega)$ owned by the household at $\omega$ is the sum of the two assets' values.

$$
v(t, \omega)=\bar{k}(t, \omega)+a(t, \omega)
$$

The household at $\omega$ collects the following rent from the land that the household owns.

$$
\bar{r}(t, \omega)=\int_{0}^{L} R(t, \widetilde{\omega}) \bar{l}(t, \omega, \widetilde{\omega}) d \widetilde{\omega}, 0 \leq \omega \leq L
$$

The total land rent of the economy is equal to the land rent that the population owns.

$$
\int_{0}^{L} \bar{r}(t, \omega) d \omega=\int_{0}^{L} R(t, \omega) d \omega, 0 \leq \omega \leq L
$$

The household at $\omega$ has the following current income:

$$
y(t, \omega)=r^{*} \bar{k}(t, \omega)+w(t)+\bar{r}(t, \omega), \quad 0 \leq \omega \leq L
$$

from the interest payment $r^{*}(t) k(t)$, and the wage payment $w(t)$, and the land rent income $\bar{r}(t)$. The disposable income $\hat{y}(t, \omega)$ is the sum of the current income and the total value of wealth.

$$
\hat{y}(t, \omega)=y(t, \omega)+v(t, \omega)
$$

\section{(g) The Budget}

At each point in time, the household at location $\omega$ distributes the total available budget between housing $I(t, \omega)$, savings $s(t, \omega)$, consumption of industrial goods $c(t, \omega)$, and travelling $c_{T}(\omega)$. The total expenditure is:

$$
R(t, \omega) l(t, \omega)+c(t, \omega)+s(t, \omega)+c_{T}(t, \omega), 0 \leq \omega \leq L
$$

The disposable income equals the total expenditure, i.e.

$$
R(t, \omega) l(t, \omega)+c(t, \omega)+s(t, \omega)+c_{T}(t, \omega)=\hat{y}(t, \omega), 0 \leq \omega \leq L
$$

Inserting (10) and (11) in (12), we get:

$$
R(t, \omega) l(t, \omega)+c(t, \omega)+s(t, \omega)=\bar{y}(t, \omega)
$$

where

$$
\bar{y}(t, \omega) \equiv\left(1+r^{*}(t)\right) \bar{k}(t, \omega)+w(t)+\bar{r}(t, \omega)+a(t, \omega)-c_{T}(t, \omega)
$$


(h) Utility, Amenity and Optimal Solution

We assume that utility level $U(t, \omega)$ of the household at location $\omega$ is dependent on $T(\omega), I(t, \omega), s(t, \omega)$, and $c(t, \omega)$ as follows:

$$
\begin{aligned}
U(t, \omega)= & \theta(t, \omega) T^{\sigma_{0}(t)}(\omega) c^{\xi_{0}(t)}(t, \omega) l^{\eta_{0}(t)}(t, \omega) s^{\lambda_{0}(t)}(t, \omega), \\
& \sigma_{0}(t), \xi_{0}(t), \eta_{0}(t), \quad \lambda_{0}(t)>0
\end{aligned}
$$

in which $\sigma_{0}(t), \xi_{0}(t), \eta_{0}(t)$ and $\lambda_{0}(t)$ are propensities to use leisure time, to consume goods, to consume housing, and to hold wealth, respectively. The amenity $\theta(\omega, t)$ at $\omega$ is specified as follows:

$$
\theta(t, \omega)=\theta_{1}(t) n^{\mu(t)}(t, \omega), \quad \theta_{1}(t)>0
$$

Maximising $U(t, \omega)$ subject to (8) yields:

$$
l(t, \omega)=\frac{\eta(t) \bar{y}(t, \omega)}{R(t, \omega)}, c(t, \omega)=\xi(t) \bar{y}(t, \omega), s(t, \omega)=\lambda(t) \bar{y}(t, \omega)
$$

where

$$
\begin{aligned}
\eta(t) & \equiv \rho(t) \eta_{0}(t), \quad \xi(t) \equiv \rho(t) \xi_{0}(t), \lambda(t) \equiv \rho(t) \lambda_{0}(t) \\
\rho(t) & \equiv \frac{1}{\eta_{0}(t)+\xi_{0}(t)+\lambda_{0}(t)}
\end{aligned}
$$

\section{(i) Equal Utility Level over the Residential Area}

The assumption is that households get the same level of utility at any location at any point. As the city is small, its utility level is determined in the national economy. As we assume free and costless movement of people, we have:

$$
U(t, \omega)=U^{*}(t), \quad 0 \leq \omega \leq L
$$

\section{(j) Wealth Accumulation}

According to the definition of $s(t, \omega)$, the wealth accumulation of the household at location $\omega$ is given by:

$$
\dot{v}(t, \omega)=s(t, \omega)-v(t, \omega), 0 \leq \omega \leq L
$$

(k) The Land Market Equilibrium

According to the definition, total value of the urban land $V(t)$ is:

$$
V(t)=\int_{0}^{L} p(t, \omega) d \omega
$$

The city's total value of land owned by the urban residents is:

$$
V^{*}(t)=\int_{0}^{L} n(t, \omega) a(t, \omega) d \omega
$$


Equality of the two values implies:

$$
\int_{0}^{L} n(t, \omega) a(t, \omega) d \omega=\int_{0}^{L} p(t, \omega) d \omega
$$

(I) The Equilibrium for Goods Production and Consumption

The total consumption $C(t)$ is:

$$
C(t)=\int_{0}^{L} n(t, \omega) c(t, \omega) d \omega
$$

Next, we built the spatial growth model.

\section{The Spatial Dynamics}

This section examines properties of the spatial model. The following lemma provides a computational procedure to plot the motion of the economic system.

\section{Lemma}

The dynamics of wealth per household $v(t)$ is described by the following differential equation:

$$
\dot{v}(t)=\Omega(v(t))
$$

in which $\Omega$ is a function of $v(t)$ defined in the appendix. For a given $v(t)$, we uniquely determine all the other variables by the following procedure: $w(t)$ by $(4) \rightarrow \bar{Y}(t)$ by $(\mathrm{A} 3) \rightarrow c(t)$ by $(18) \rightarrow s(t)$ by (18) $\rightarrow n(\omega)$ by (A16) $\rightarrow N(t)$ by (A17) $\rightarrow I(\omega)$ by (5) $\rightarrow R(t, \omega)$ by $(\mathrm{A} 5) \rightarrow p(t, \omega)$ by $(6) \rightarrow U(t, \omega)$ by $(\mathrm{A} 14) \rightarrow \tilde{v}(t)=\int_{0}^{L} n(\omega) v(\omega) d \omega \rightarrow \tilde{r}(t)=$ $\int_{0}^{L} R(\omega) d \omega \rightarrow K(t)$ by $(4) \rightarrow F(t)$ by $(2) \rightarrow \bar{K}(\mathrm{t})$ by $(\mathrm{A} 9) \rightarrow C(t)$ by $(\mathrm{A} 12)$.

In the lemma we define the total wealth $\tilde{v}(t)$ by:

$$
\tilde{v}(t) \equiv \int_{0}^{L} n(t, \omega) v(t, \omega) d \omega
$$

First, we examine the case that all the parameters are constant and are specified as in Zhang (2017):

$$
\begin{aligned}
& r^{*}=0.05, U^{*}=7, \alpha=0.45, A=0.8, \quad N=50, L=1, \\
& T_{0}=1, \delta_{k}=0.03, \lambda_{0}=0.8, \sigma_{0}=0.2, \xi_{0}=0.1, \\
& \eta_{0}=0.02, \quad \bar{c}=0.01, \theta_{1}=1, \mu=-0.05
\end{aligned}
$$

The initial condition is: $v(0)=15$. Under $(26)$, we have $w=1.51$. Figure 1 shows all the other variables over time and space. 

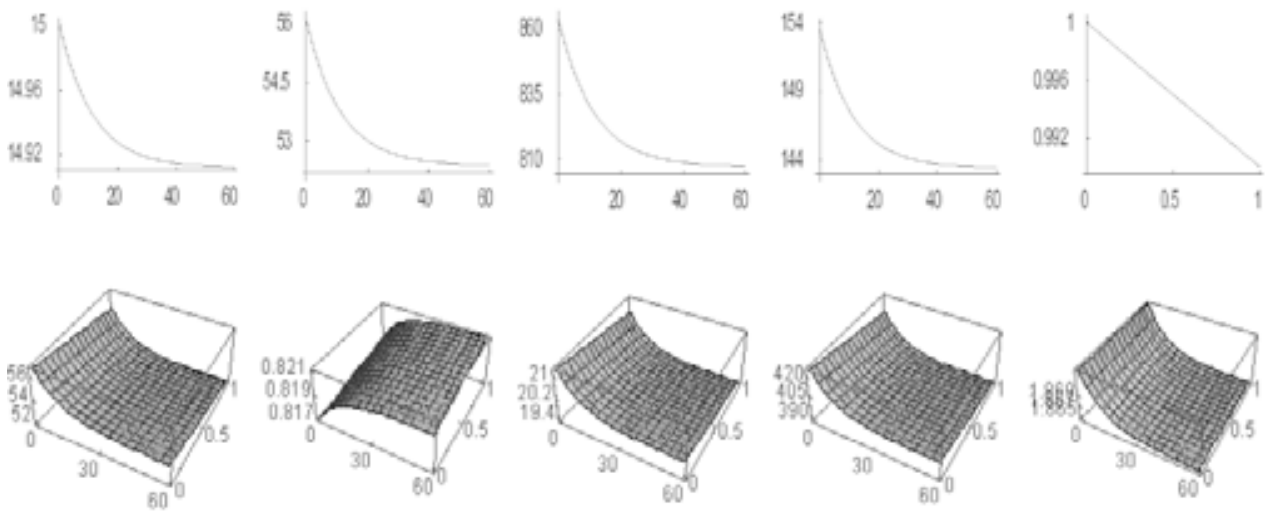

Figure 1. The motion of the economy over space

\section{Comparative Dynamic Analysis}

This section carries out comparative dynamic analysis with regards to some parameters. We introduce a symbol $\bar{\Delta} x$ to stand for the rate of change of the variable $x$ in percentage due to changes in the value of a parameter.

\subsection{Periodic Oscillations in the National Utility}

First, we examine the case that the national utility is oscillatory as follows:

$$
U^{*}(t)=7+0.01 \sin (t)
$$

The simulation results are plotted in Figure 2.
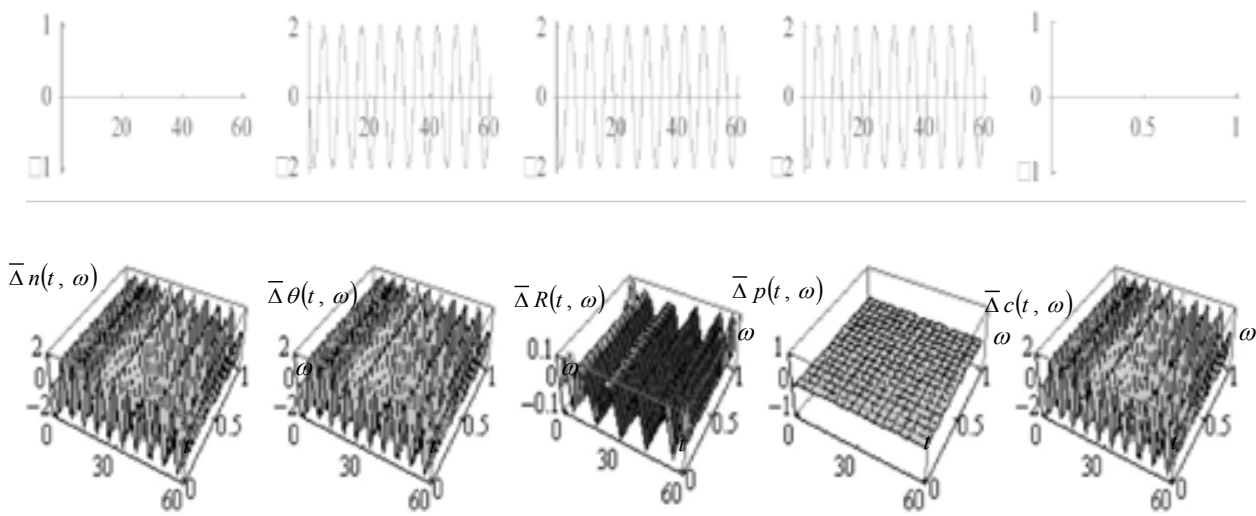

Figure 2. Exogenous periodic shocks in the national utility 


\subsection{Periodic Fluctuations in the Rate of Interest}

We now allow the following periodic fluctuations in the rate of interest:

$$
r^{*}(t)=0.05+0.005 \sin (t)
$$

The changes in the variables are plotted in Figure 3.
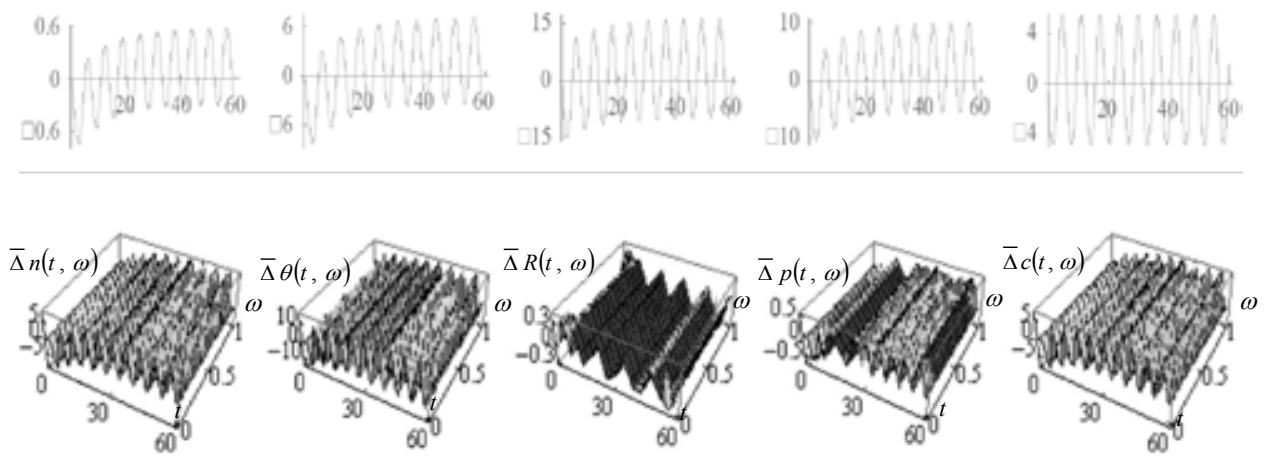

Figure 3. Exogenous periodic shocks in the rate of interest

\subsection{Periodic Fluctuations in Total Factor Productivity}

We now examine the effects of the following periodic fluctuations in the total factor productivity:

$$
A(t)=0.8+0.01 \sin (t)
$$

The simulation results on the variables are plotted as in Figure 4.
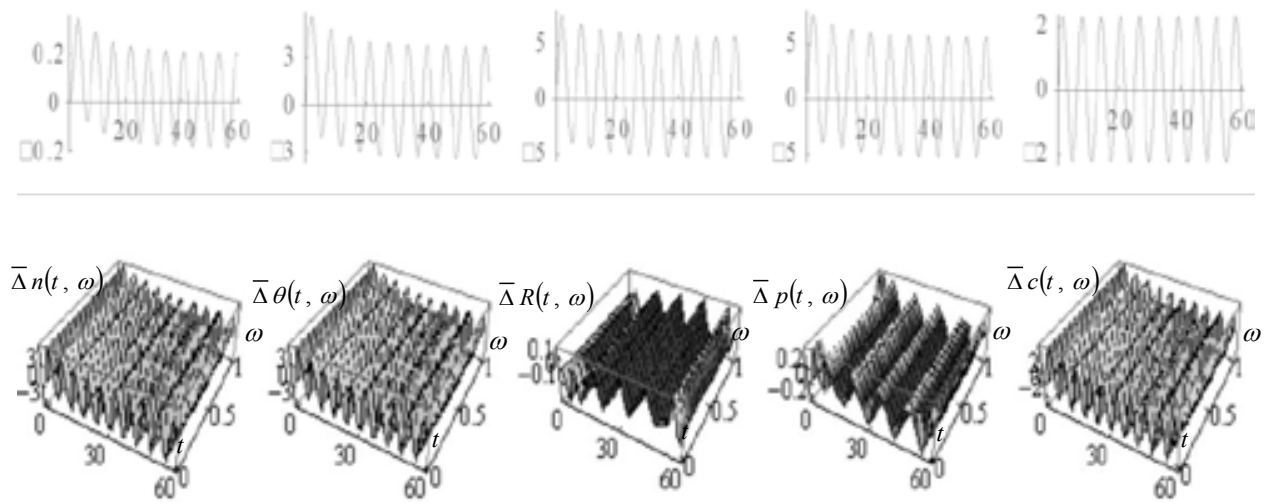

Figure 4. Exogenous periodic shocks in total factor productivity 


\section{Concluding Remarks}

The unique contribution of the paper is that the model not only integrates the Solow growth and Alonso residential distribution models with endogenous land value for a small urban economy, it also explores issues related to existence of business cycles and determinants of business cycles. It is a contribution to the literature of spatial economic growth by identifying existence of exogenous cycles in an urban growth model built on microeconomic foundation. In fact, there are a few spatial models of economic growth built on microeconomic foundation. Most of the urban economic models are static in the literature of urban economics. Although much of the basic model in this study is based on Zhang's model, the analysis is not so simple as all the parameters are time dependent. As the model is based on a few key models in economic literature and each of these key models have been generalised and extended in different ways, we can extend the model along these lines. A challenge for future research is to address issues about endogenous expectations on prices.

\section{References}

Alonso, W. (1964). Location and land use: Toward a general theory of land rent. Cambridge, MA: Harvard University Press.

Chiarella, C., \& Flaschel, P. (2000). The dynamics of Keynesian monetary growth: Macro foundations. Cambridge, UK: Cambridge University Press.

Gandolfo, G. (2005). Economic dynamics. Berlin: Springer-Verlag.

Lorenz, H.W. (1993). Nonlinear dynamical economics and chaotic motion. Berlin: Springer-Verlag.

Puu, T. (2011). Nonlinear economic dynamics. Berlin: Springer-Verlag.

Shone, R. (2002). Economic dynamics - Phase diagrams and their economic application. Cambridge, UK: Cambridge University Press.

Solow, R. (1956). A contribution to the theory of growth. Quarterly Journal of Economics, 70(1), 65-94. https://doi.org/10.2307/1884513

Zhang, W.B. (1991). Synergetic economics. Heidelberg: Springer-Verlag.

Zhang, W.B. (2005). Differential equations, bifurcations, and chaos in economics. Singapore: World Scientific.

Zhang, W.B. (2006). Discrete dynamical systems, bifurcations and chaos in economics. Amsterdam: Elsevier.

Zhang, W.B. (2017). Economic growth, land value, and land rent with residential location in a small city. Conference Proceedings, 2017 International Symposium on Economics and Social Science - Summer Session Conference, 23-25 August 2017, Sapporo, Japan. 


\section{Appendix}

Insert the definition of $\bar{r}(\omega)$ in the definition of $\bar{y}$ :

$$
\begin{aligned}
\bar{y}(\omega)= & \left(1+r^{*}\right) \bar{k}(\omega)+w+\int_{0}^{L} R(\widetilde{\omega}) \bar{l}(\omega, \widetilde{\omega}) d \widetilde{\omega}+ \\
& \int_{0}^{L} p(\widetilde{\omega}) \bar{l}(\omega, \widetilde{\omega}) d \widetilde{\omega}-c_{T}
\end{aligned}
$$

Insert (6) in (A1):

$$
\bar{y}(\omega)=\left(1+r^{*}\right) \bar{k}(\omega)+w+\left(1+r^{*}\right) \int_{0}^{L} p(\widetilde{\omega}) \bar{l}(\omega, \widetilde{\omega}) d \widetilde{\omega}-c_{T}
$$

Insert (9) and (10) in (A2):

$$
\bar{y}(\omega)=\left(1+r^{*}\right) v(\omega)+w-c_{T}
$$

From (18) and (A3), we have:

$$
R(\omega) l(\omega)=(1+r) \eta v(\omega)+\eta w-\eta c_{T}
$$

Insert (5) in (A4):

$$
R(\omega)=\left(1+r^{*}\right) \eta n(\omega) v(\omega)+\eta n(\omega) w-\eta n(\omega) c_{T}
$$

Integrate (A5) from 0 to $L$ :

$$
\tilde{r}=\left(1+r^{*}\right) \eta \tilde{v}+\eta w N-\eta c_{T} N
$$

where we use (12) and

$$
\tilde{r} \equiv \int_{0}^{L} R(\omega) d \omega, \quad \tilde{v} \equiv \int_{0}^{L} n(\omega) v(\omega) d \omega
$$

From the definitions of $v$ we have:

$$
\tilde{v}=\int_{0}^{L}(n(\omega) \bar{k}(\omega)+n(\omega) a(\omega)) d \omega=\bar{K}+V^{*}
$$

where $\bar{K} \equiv \int_{0}^{L} n(\omega) \bar{k}(\omega) d \omega$. From (6) we have:

$$
\tilde{r}=r V^{*}
$$

From (9) and (10) we have:

$$
\tilde{v}=\bar{K}+\frac{\tilde{r}}{r^{*}}
$$


From (A6) and (A9) we solve:

$$
\tilde{v}=\hat{r}\left(r^{*} \bar{K}+\eta w N-\eta c_{T} N\right)
$$

where

$$
\hat{r}=\frac{1}{r^{*}-\left(1+r^{*}\right) \eta}
$$

From (18) and (A3), we have:

$$
c=\left(1+r^{*}\right) \xi v+\xi w-\xi c_{T}
$$

Multiplying the two sides of (A11) by $n$ and then integrate the resulting equation from 0 to $L$ :

$$
C=\left(1+r^{*}\right) \xi \tilde{v}+\xi w N-\xi c_{T} N
$$

Insert (18) and (A4) in (20):

$$
\dot{v}=\Omega(v) \equiv-\bar{r} v+\lambda w-\lambda c_{T}
$$

where $\bar{r} \equiv 1-\lambda-\lambda r^{*}$. This is a first-order linear differential equation with constant coefficients. We denote its solution by $v(\omega, t)$. In the rest of the appendix we treat $v(\omega, t)$ as known functions of time. Insert (17), (18) and (4) in (16):

$$
U(\omega)=\theta_{0} n^{\mu-\eta_{0}}(\omega) T^{\sigma_{0}}(\omega) \bar{y}^{\xi_{0}+\lambda_{0}}(\omega)
$$

where $\theta_{0} \equiv \theta_{1} \lambda^{\lambda_{0}} \xi^{\xi_{0}}$. Insert (A14) in (19):

$$
\theta_{0} n^{\mu-\eta_{0}}(\omega) T^{\sigma_{0}}(\omega) \bar{y}^{\xi_{0}+\lambda_{0}}(\omega)=U^{*}
$$

From (A15) we solve:

$$
n(\omega, t)=g(\omega, t), \quad 0 \leq \omega \leq L
$$

where

$$
g(\omega, t) \equiv\left(\frac{U^{*}}{\theta_{0} T^{\sigma_{0}}(\omega) \bar{y}^{\xi_{0}+\lambda_{0}}(\omega)}\right)^{1 /\left(\mu-\eta_{0}\right)}
$$

Insert (A16) in (1):

$$
N(t)=\int_{0}^{L} g(\omega, t) d \omega
$$

We can now determine all the variables over time and space by the procedure in the lemma. 
